\title{
VALORES DE REFERÊNCIA DO DRIS PARA A SOJA, CULTIVARES EMBRAPA 59 E BR 37, EM CARAMBEÍ - PARANÁ
}

\author{
REFERENCE VALUES OF DRIS FOR THE SOYBEAN, cV. EMBRAPA \\ 59 AND BR 37, IN CARAMBEÍ AT PARANÁ STATE, BRAZIL
}

\author{
Shizuo MAEDA ${ }^{1}$ \\ Pedro RONZELLI Júnior ${ }^{2}$
}

\begin{abstract}
RESUMO
As análises de solo e de folhas, cujos resultados são interpretados com base em faixas de concentração, são os critérios mais utilizados para a caracterização nutricional da cultura da soja no Brasil. A interpretação de resultados analíticos de forma isolada para cada nutriente e sua não hierarquização quanto as limitações nutricionais, bem como possíveis efeitos de fatores como variedade, idade de tecido e edafoclimáticos locais, diminuem eficiência de tais critérios. O Sistema Integrado de Diagnose e Recomendação (DRIS) surge como alternativa aos referidos critérios interpretativos. Apesar de ser menos suscetível aos problemas que interferem nos critérios mencionados, o DRIS também é influenciado pelos mesmos fatores. Tais efeitos podem se minimizados por meio do desenvolvimento de "valores de referência" particularizados para os fatores que interferem em suas magnitudes e portanto em sua eficiência. Com o objetivo de obter valores de referência do DRIS para soja em Carambeí, PR, amostras de folhas e grãos foram coletadas em lavouras comerciais na safra 1998/99. Tais amostras foram coletadas em glebas com as cultivares Embrapa 59 e BR 37 manejadas no sistema plantio direto na palha (SPD) e com a cultivar Embrapa 59 manejada no sistema de plantio convencional (SPC). Os resultados analíticos das amostras de folhas e as correspondentes produtividades de grãos formaram a base de dados para 0 desenvolvimento do método DRIS. Os valores de referência obtidos a partir das amostras da cultivar Embrapa 59 no SPD e SPC e da cultivar BR 37 no SPC proporcionam estimativas diferentes para os índices de diagnose do DRIS resultando em diagnósticos distintos.

Palavras-chave: Glycine max, nutrição mineral, DRIS, Carambeí - Paraná/Brasil.
\end{abstract}

\begin{abstract}
Soil and leaves analysis to characterize the nutritional state of crops are usually interpreted on basis of optimal concentration ranges. This approach has been widely used for nutritional characterization of soybean in Brazil. However, the interpretation of analytical results for each nutrient in an isolated way without considering any hierarchy about nutritional limitations as well as possible effects of variety, sample age and local soil and climatic conditions has restricted its efficiency. To overcome part of these problems, the Diagnosis and Recommendation Integrated System (DRIS) appears as an alternative approach to the usual interpretation of nutritional state based on optimal concentration ranges. DRIS is also influenced by the same factors (i.e. variety, sample age etc) as for the concentrations range approach but in a lower degree. Such effects can be minimized by means of "reference values", which are particularized to the factors that interfere in its magnitude and, therefore, in its efficiency. With the aim to obtain reference values of DRIS for soybeans in Carambeí, at Paraná state, Brazil, samples of leaves and grains were collected in areas of commercial soybeans crops during the growing season 1998-1999. The soybeans crops were cultivated using Embrapa 59 variety in a no-till system (SPD) and in a conventionaltill system (SPC), and also BR 37 variety in a SPD. Results from leaf samples and productivity of grains formed the database for the development of DRIS approach. The reference values obtained from Embrapa 59 and BR 37 varieties using SPD and SPC and from Embrapa 59 using SPC gave different estimations for the DRIS diagnosis indexes and, therefore, resulting in different diagnoses.

Key- words: Glycine max, mineral nutrition, DRIS, Carambeí - Paraná/Brasil.
\end{abstract}

\footnotetext{
${ }^{1}$ Eng $^{\circ}$ Agroํ․ Doutor, CREA 14.915/D, 7ª Região, Pesquisador da EMBRAPA Florestas. Estrada da Ribeira, km 111, CP 319 , Colombo (PR), CEP 83411-000. E-mail: maeda@cnpf.embrapa.br.

${ }^{2}$ Eng $^{\circ}$ Agr $^{\circ}$, Doutor, CREA 37.209/D, 6ª Região, Visto 5.867-PR, Professor Adjunto IV do Departamento de Fitotecnia e Fitossanitarismo da Universidade Federal do Paraná.
} 


\section{INTRODUÇÃO}

A fertilidade de solos destinados ao cultivo de soja no Brasil têm sido avaliada por meio das análises de solo e de folhas, sendo os resultados analíticos interpretados com base no critério das faixas de concentração. Embora para espécies como de Eucaliptus, a interpretação dos resultados analíticos venha sendo realizada com base na proporção entre nutrientes, em geral, a interpretação das concentrações foliares de nutrientes é feita de forma independente uns dos outros, o que se constitui numa limitação do método. Assim, os resultados analíticos são interpretados sem considerar as interações existentes entre os distintos nutrientes. Além disso, quando da utilização desse critério e quando dois ou mais nutrientes estão abaixo de suas faixas de concentração críticas, o método não permite hierarquizar essas limitações. A idade do tecido analisado e variações nas concentrações de nutrientes entre cultivares também limitam a diagnose nutricional por esse critério.

As deficiências apresentadas pelo critério das faixas de concentração podem ser minimizadas pelo Sistema Integrado de Diagnose e Recomendação DRIS (BEAUFILS, 1973). Estudos realizados com esse método, em diferentes culturas, têm indicado boas perspectivas para a diagnose nutricional (LEANDRO, 1998, BEVERLY et al., 1986 e SUMMER, 1979). O DRIS é um sistema de cálculo no qual razões de relações entre concentrações de nutrientes em uma amostra são comparados matematicamente com valores "ótimos" (valores de referência ou normas) das mesmas razões de uma população de alta produtividade (população de referência) assumida como nutricionalmente equilibrada. Essa comparação possibilita a obtenção de índices de diagnose utilizados no ordenamento dos desequilíbrios nutricionais. Inicialmente considerados como de aplicação universal, quando obtidos a partir de informações coletadas em regiões com ampla diversidade ambiental, os valores de referência do DRIS têm mostrado efeito de fatores edafoclimáticos regionais, resultando em diagnósticos errôneos quando tais valores são obtidos em condições distintas daquelas da amostra sob diagnose. Isto indica a necessidade de se obter valores de referência particularizados para os fatores que neles interferem para o incremento da eficiência do DRIS (BEVERLY et al.,1986 e BEAUFILS, 1973).

Assim, o objetivo deste trabalho foi o de obter valores de referência para o DRIS para a soja cultivada na região de Carambeí - Paraná.

\section{METODOLOGIA}

Para o desenvolvimento do trabalho, amostras de folhas e de grãos foram coletadas na safra 1998/ 1999, na Fazenda FrankAnna, localizada no município de Carambeí - Paraná. As amostras foram coletadas em lavouras manejadas no sistemas de plantio direto na palha (SPD) e convencional (SPC) exploradas com a cultivar Embrapa 59 e com a cultivar BR 37 em SPD. Oitenta amostras foram coletadas em cada uma das áreas selecionadas, sendo que as de folhas foi realizada no estádio fenológico R1 (COSTA e MARQUEZAN, 1982) de acordo com o recomendado por Martinez et al. (1999), Borkert et al. (1994) e Bataglia e Dechen (1986). Por sua vez, as amostras de grãos, para a determinação da produtividade, foram coletadas no estádio R8 na escala de Costa e Marquezan (1982). Nas amostras de folhas foram determinados os teores totais de N, P, K, Ca, Mg, S, B, Cu, Fe, Mn e Zn, conforme metodologia descrita em Malavolta et al. (1997).

O desenvolvimento do DRIS foi feito conforme os procedimentos sugeridos por Schultz e Villiers (1987).

\section{Formação da base de dados}

A base de dados utilizada para o desenvolvimento dos valores de referência do DRIS da cultivar Embrapa 59 no SPD e no SPC e da BR 37 no SPD, foi composta por 80 amostras cada. Também foi formada uma base de dados pelo agrupamento de todas as informações das três bases de dados mencionadas anteriormente. As informações utilizadas para a formação da base de dados e para o desenvolvimento do DRIS foram os teores totais de nutrientes nas folhas e a produtividade de grãos.

\section{Seleção da população de referência}

Cada base de dados, foi dividida em duas subpopulações considerando-se a produtividade de 4.000 $\mathrm{kg} \cdot \mathrm{ha}^{-1}$ como o limite entre a sub-população A, de alta produtividade $\left({ }^{3} 4.000 \mathrm{~kg} \cdot \mathrm{ha}^{-1}\right)$ e a sub-população $B$, de baixa produtividade $\left(<4.000 \mathrm{~kg} \cdot \mathrm{ha}^{-1}\right)$.

\section{Obtenção dos valores de referência}

Em cada sub-população, de cada base de dados, os nutrientes foram expressos em todas as relações possíveis na forma de quociente e, em seguida, foram calculadas as médias $\left(X_{A}\right.$ e $\left.X_{B}\right)$, os desvios padrão $\left(S_{A}\right.$ e $\left.S_{B}\right)$, os coeficientes de variação (CV) e as variâncias $\left(S_{A}{ }^{2}\right.$ e $\left._{B}{ }^{2}\right)$ da concentração de cada nutriente e dos valores dos quocientes das relações entre estes, de acordo com Walworth e Summer (1987) e Beaufils (1973). Pela comparação da razão entre as variâncias das relações nutricionais das duas sub-populações $\left(\mathrm{s}_{\mathrm{B}}{ }^{2} /\right.$ $\mathrm{S}_{\mathrm{A}}{ }^{2}$ ), para cada relação nutricional, na forma direta e inversa (exemplo N/P e P/N), foram selecionadas aquelas com maior razão entre as variâncias, conforme Walworth e Sumner (1987). As médias dos quocientes das relações entre as concentrações dos nutrientes selecionados e os seus respectivos coeficientes de variação, correspondentes à sub- 
MAEDA, S.; RONZELLI Jr., P. Valores de referência do dris para...

população de alta produtividade $(A)$, representam os valores de referência do DRIS de cada uma das bases de dados formadas.

Os valores de referência desenvolvidos com as informações obtidas para a cultivar Embrapa 59 no SPD e no SPC e para a cultivar BR 37 no SPD foram comparados entre si e com aqueles obtidos com o agrupamento de todas as informações das três bases de dados mencionadas anteriormente. A comparação entre os valores de referência foi feita pelo teste $\mathrm{t}$.

\section{Cálculo dos índices de diagnose do DRIS para comparação entre os valores de referência}

As amostras com produtividades inferiores a $4.000 \mathrm{~kg} \cdot$ ha $^{-1}$ foram submetidas à diagnose pelo DRIS, com base nos valores de referência da cultivar Embrapa 59 no SPD e SPC e da cultivar BR 37 no SPD visando comparar os índices de diagnose do DRIS. O cálculo do índice de diagnose do DRIS, para cada nutriente, foi feito por intermédio da fórmula geral proposta por Beaufils (1973), conforme a Equação 1:

Equação (1)

ÍndiceX $=\left[\frac{f(X / Y 1)+\ldots+f(X / Y m)-f(Z 1 / X)-\ldots-f(Z n / X)}{m+n}\right]$

sendo:

$X=$ nutriente genérico sob avaliação;

$\mathrm{Y} 1, \mathrm{Y} 2$ e $\mathrm{Yn}=$ nutrientes genéricos que aparecem no denominador das relações com 0 nutriente genérico $X$;

$\mathrm{Z1}, \mathrm{Z2}$ e $\mathrm{Zn}=$ nutrientes genéricos que aparecem no numerador das relações com o nutriente genérico $X$;

$\mathrm{m}$ = número de funções em que o nutriente em processamento se encontra no numerador da relação; $\mathrm{n}=$ número de funções em que o nutriente em processamento se encontra no denominador da relação;

$f(X / Y 1)=$ função da relação entre os nutrientes genéricos $\mathrm{X}$ e $\mathrm{Y} 1 \mathrm{e}$,

$f(Z 1 / X)=$ função da relação entre os nutrientes genéricos $Z 1$ e $X$.

Por outro lado, o cálculo das funções foi feito conforme Elwali e Gascho (1984):

Equação (2)

$$
f(X / Y)=\left[\left(\frac{X / Y}{x / y}\right)-1\right]\left(\frac{100 k}{C V}\right) \quad \text { se } X / Y>x / y+s_{x / y}
$$

Equação (3)

$$
f(X / Y)=\left[1-\left(\frac{x / y}{X / Y}\right)\right]\left(\frac{100 k}{C V}\right) \text { se } \mathrm{X} / \mathrm{Y}<\mathrm{x} / \mathrm{y}-\mathrm{s}_{\mathrm{x} / \mathrm{y}}
$$

Equação (4)

$$
f(X / Y)=0 \quad \text { se } x / y-s_{x / y} \leq X / Y £ x / y+s_{x / y}
$$

sendo:

$\mathrm{X} / \mathrm{Y}=$ valor do quociente das concentrações entre dois nutrientes genéricos $X$ e $Y$ na planta sob avaliação;

$x / y=\quad$ valor de referência entre dois nutrientes genéricos $\mathrm{X}$ e $\mathrm{Y}$;

$\mathrm{CV}=\quad$ coeficiente de variação da relação entre dois nutrientes genéricos $\mathrm{X}$ e $\mathrm{Y}$ da população de referência;

$\mathrm{k}=\quad$ coeficiente de sensibilidade, de valor arbitrário, utilizado nesta equação de acordo com
Beaufils (1973), para que os valores dos índices de diagnose apresentem magnitudes convenientes, sendo o valor adotado neste trabalho igual a 10; e $\mathrm{s}_{x / y}=$ desvio padrão da população de referência.

Os sinais das funções inseridas na fórmula de cálculo dos índices de diagnose foram atribuídos considerando-se a posição do nutriente na relação. Se no numerador o sinal é positivo e se no denominador é negativo Beaufils (1973). 
MAEDA, S.; RONZELLI Jr., P. Valores de referência do dris para...

\section{RESULTADOS E DISCUSSÃO}

As médias dos teores foliares de nutrientes da cultivar Embrapa 59 no SPC e SPD e da cultivar BR 37 no SPD, bem como dos dados agrupados, estão relacionados na Tabela 1 . Também é demonstrada a comparação entre os teores médios pelo teste $t$. Conforme pode ser observado na tabela mencionada, as médias dos teores de alguns nutrientes da cultivar Embrapa 59 no SPC apresentaram diferenças significativas a $5 \%$ de probabilidade quando comparadas em relação às médias dos teores de nutrientes da mesma cultivar obtida no SPD. Diferenças significativas também foram observadas nos teores médios de nutrientes entre a cultivar Embrapa 59 no SPD e SPC e a cultivar BR 37 no SPD. Por outro lado, quando se comparou os teores médios das cultivares Embrapa 59 no SPD e SPC com os teores médios dos dados agrupados (coluna Geral na Tabela 1) observouse que houve uma redução no número de nutrientes cujos teores foram significativamente diferentes quando comparados entre si com base nos dados de cada variedade isoladamente. Porém, quando se comparou os teores médios dos nutrientes da cultivar BR 37 no SPD, com os teores médios dos mesmos nutrientes dos dados agrupados, observou-se um aumento do número de nutrientes com diferenças significativas. As diferenças observadas nos teores dos nutrientes entre as cultivares Embrapa 59 e BR 37 no SPD, devem-se, provavelmente, às diferenças nas necessidades metabólicas e/ou na capacidade de absorção dos nutrientes das mesmas (MASCARENHAS et al., 1980 e KEOGH et al., 1972).

Quando se comparou os valores dos quocientes das relações nutricionais estabelecidas entre as concentrações foliares dos nutrientes analisados nas amostras das cultivares Embrapa 59 no SPD e SPC e BR 37 no SPD, e os da base de dados agrupados com produtividade superior a $4.000 \mathrm{~kg} \cdot \mathrm{ha}^{-1}$, observaram-se diferenças nos quocientes de grande número de relações estabelecidas (Tabela 2 ). As diferenças observadas entre os quocientes das relações nutricionais resultaram das variações nas concentrações foliares de nutrientes entre as cultivares amostradas no mesmo sistema de manejo e entre a mesma cultivar, em ambos os sistemas de manejo do solo.

TABELA 1 - Médias de teores foliares de nutrientes da cultivar Embrapa 59 no sistema de plantio direto na palha (SPD) e sistema de plantio convencional (SPC), da cultivar BR 37 no SPD e dos dados agrupados obtidos de amostras com produtividades superiores a $4.000 \mathrm{~kg} \cdot \mathrm{ha}^{-1}$, Carambeí, PR, 1998/1999.

\begin{tabular}{|c|c|c|c|c|c|c|c|c|c|c|}
\hline \multirow[t]{2}{*}{ Variável } & Embrapa 59 - SPC & $\begin{array}{c}t \\
\text { (1) }\end{array}$ & $\begin{array}{c}\mathrm{t} \\
\text { (2) }\end{array}$ & Embrapa 59 - SPD & $\begin{array}{l}t_{(3)} \\
\text { (3) }\end{array}$ & $\begin{array}{l}\mathrm{t} \\
\text { (2) }\end{array}$ & BR 37 - SPD & $\begin{array}{l}\mathrm{t}_{(4)} \\
\text { (a) }\end{array}$ & $\begin{array}{l}\mathrm{t} \\
\text { (2) }\end{array}$ & Geral \\
\hline & Média & & & Média & & & Média & & & Média \\
\hline $\mathrm{N}$ & 58,9 & * & * & 52,1 & * & * & 63,1 & & * & $\overline{56,9}$ \\
\hline$P$ & 3,6 & & & 3,5 & & & 3,6 & & & 3,5 \\
\hline K & 20,3 & * & & 21,8 & * & & 19,5 & & & 20,8 \\
\hline $\mathrm{Ca}$ & 7,5 & * & & 6,6 & * & * & 8,5 & * & * & 7,3 \\
\hline $\mathrm{Mg}$ & 3,0 & & & 2,8 & * & * & 3,4 & * & * & 3,0 \\
\hline $\mathrm{S}$ & 2,7 & & & 2,5 & * & & 2,6 & & & 2,6 \\
\hline B & 85,4 & * & * & 62,3 & * & & 27,9 & * & * & 65,8 \\
\hline $\mathrm{Cu}$ & 9,3 & & & 10,4 & & & 11,4 & * & * & 10,1 \\
\hline $\mathrm{Fe}$ & 87,0 & * & * & 72,9 & * & * & 82,3 & & & 79,9 \\
\hline $\mathrm{Mn}$ & 35,0 & * & & 28,8 & * & * & 48,4 & * & * & 34,4 \\
\hline $\mathrm{Zn}$ & 38,4 & * & * & 33,6 & * & & 30,8 & * & * & 35,0 \\
\hline
\end{tabular}

(1) Comparação de Embrapa 59 - SPC com Embrapa 59 - SPD,

(2) Comparação em relação à média dos dados agrupados,

(3) Comparação de Embrapa 59 - SPD com BR 37 - SPD,

(4) Comparação de Embrapa 59 - SPC com BR 37 - SPD, e

* - significativo a $5 \%$ de probabilidade pelo teste $t$

Diferenças nos teores de nutrientes e portanto nos valores de referência (VR) entre regiões geográficas, não são necessariamente indicações das necessidades nutricionais das plantas, e sim meramente o reflexo dos efeitos do solo e do clima sobre a condição nutricional das mesmas (BEVERLY et al. 1986 e WALWORTH et al. 1988). Á exceção do $\mathrm{N}$ e do $\mathrm{B}$, os teores dos demais nutrientes enquadraram-se nas respectivas faixas de suficiência (EMBRAPA SOJA, 1999). Os teores de $\mathrm{N}$ e de $\mathrm{B}$ na cultivar Embrapa 59, respectivamente no SPC e SPD, enquadraram-se na faixa de teor alto e o teor de $B$ na mesma cultivar, no SPC, na faixa de teor excessivo (EMBRAPA SOJA, 1999).

O agrupamento das amostras teve como objetivo aumentar a base de dados visando atender as sugestões apresentadas por diversos autores (WALWORTH e SUMNER, 1987, SCHUTZ e VILLIERS, 1987, SUMNER, 1979 e BEAUFILS, 1973). No entanto, conforme pode ser observado na coluna Geral da Tabela 2, o número de relações nutricionais significativamente diferentes entre aquelas das cultivares amostradas e as relações da base de dados agrupada foi elevado. 
MAEDA, S.; RONZELLI Jr., P. Valores de referência do dris para...

TABELA 2 - Quantidade de relações nutricionais que apresentaram diferenças significativas - teste t a $5 \%$ de probabilidade - relativas entre si e em cada base de dados e em relação à base de dados global considerando todas as relações nutricionais estabelecidas (110 relações), obtidas a partir de amostras coletadas em Carambeí, PR, 1998/1999.

\begin{tabular}{|c|c|c|c|c|}
\hline \multirow{2}{*}{ Base de dados } & \multicolumn{4}{|c|}{ Base de dados } \\
\hline & $\mathrm{BR} 37-\mathrm{SPD}$ & Embrapa 59-SPD & Embrapa 59 - SPC & Geral \\
\hline $\begin{array}{l}\mathrm{ER} 37-\mathrm{SPD} \\
\text { Embrapa } 59-\mathrm{SPD}\end{array}$ & - & $\begin{array}{c}80 \\
-\end{array}$ & $\begin{array}{l}50 \\
36\end{array}$ & $\begin{array}{l}77 \\
47\end{array}$ \\
\hline Embrapa 59- SPC & - & - & - & 51 \\
\hline
\end{tabular}

TABELA 3 - Frequência de ocorrência de índices de diagnose do DRIS positivos (+), negativos (-), de maior índice (>) e de menor índice (<), de nutrientes em dez amostras de folhas de áreas com produtividade de grãos menor que $4.000 \mathrm{~kg}$.ha- ${ }^{-1}$ das cultivares BR 37 (A) e Embrapa 59 em sistemas de plantio direto (B) e da Embrapa 59 em sistema convencional (C), estimadas de acordo com valores de referência do DRIS desenvolvidos com amostras de cada uma das cultivares, nos sistemas mencionados e, com as bases de dados agrupadas (D), obtidos de amostras coletadas em Carambeí, PR, 1998/1999.

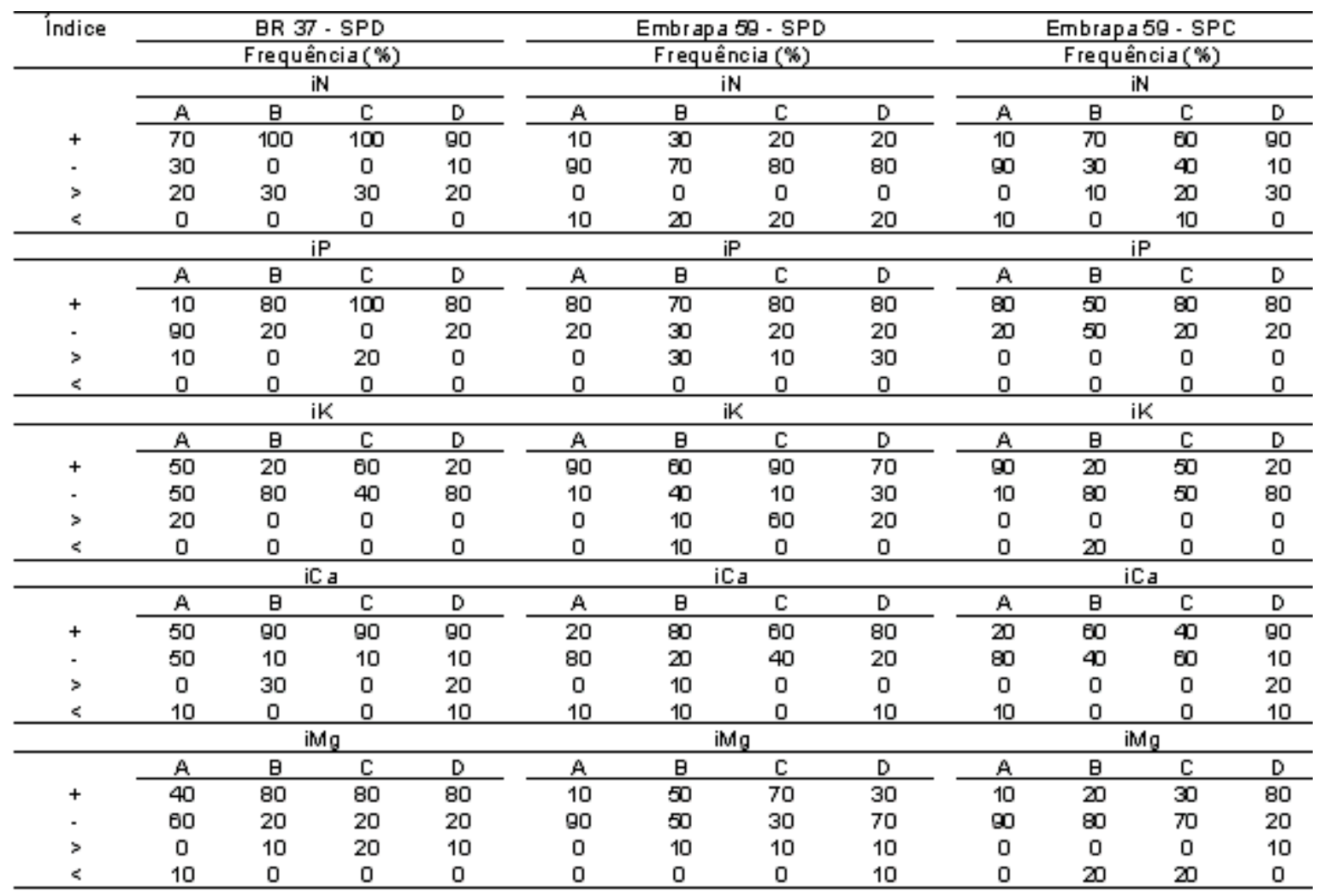

Mais importante que as diferenças observadas entre os VR estabelecidos sob diferentes condições edáficas é a interpretação dos índices de diagnose do DRIS de cada nutriente sob análise. Na Tabelas 3 são descritas as freqüências de ocorrência de índices de diagnose do DRIS positivos, negativos, de maiores e de menores valores para os macronutrientes analisados em 10 amostras com produtividades inferiores a $4.000 \mathrm{~kg} \cdot \mathrm{ha}^{-1}$, de cada uma das cultivares amostradas em ambos os sistemas de manejo do solo no caso da cultivar Embrapa 59 e no SPD no caso da cultivar BR 37. Os mencionados índices de diagnose do DRIS foram estimados com base nos VR de cada uma das cultivares e também com base nos VR obtidos da base de dados cujas amostras foram agrupadas. Tais valores encontram-se relacionados nas Tabelas 4, 5, 6 e 7 .
Como pode ser observado na Tabela 3, a freqüência de ocorrência de índices de diagnose do DRIS positivos, negativos e de maiores e menores valores é alterada em todos os nutrientes analisados quando os índices de diagnose são estimados com base em VR do DRIS obtidos das diferentes base de dados. Esses resultados demonstram que os índices de diagnose do DRIS, calculados a partir de VR obtidos em condições edafoclimáticas, de manejo e outros fatores diferentes daquelas de uma determinada amostra sob diagnose, podem apresentar valores diferentes para os índices de diagnose e, portanto, o diagnóstico poderá ser errôneo, reforçando a necessidade de se obter VR regionalizados e particularizados para os fatores que 0 influenciam e por consequência a diagnose (BEAUFILS, 1973, BEVERLY et al., 1986 e LEANDRO, 1998). 
MAEDA, S.; RONZELLI Jr., P. Valores de referência do dris para...

TABELA 4 - Valores de referência do DRIS (média e coeficiente de variação - CV) obtidos de amostras foliares e de grãos da cultivar BR 37 coletadas em área manejada no sistema plantio direto na palha em Carambeí, PR.

\begin{tabular}{|c|c|c|c|c|c|c|c|c|}
\hline $\begin{array}{c}\text { Relação } \\
\text { nutricional }\end{array}$ & Média & $C V-\%$ & $\begin{array}{c}\text { Relaçẫo } \\
\text { nutricional }\end{array}$ & Média & $C V-\%$ & $\begin{array}{c}\text { Relaçẫo } \\
\text { nutricional }\end{array}$ & Média & $C V-\%$ \\
\hline NP & 17,51 & 12,47 & Caik & 0,44 & 15,01 & Feimg & 24,99 & 33,32 \\
\hline NKK & 3,32 & 17,72 & KIMg & 5,82 & 14,33 & $\mathrm{Mg} / \mathrm{Mn}$ & 0,08 & 40,60 \\
\hline $\mathrm{NiCa}$ & 7,72 & 21,18 & $\mathrm{~K} / \mathrm{S}$ & 7,39 & 16,69 & Znimg & 9,32 & 25,11 \\
\hline NiMg & 19,22 & 19,52 & $B i K$ & 1,49 & 39,07 & Eis & 10,69 & 34,52 \\
\hline NS & 23,98 & 10,86 & Cuik & 0,60 & 26,95 & Cu'S & 4,31 & 17,13 \\
\hline NE & 2,49 & 33,05 & Felk & 4,33 & 32,86 & Fels & 31,07 & 26,22 \\
\hline $\mathrm{NCu}$ & 5,71 & 18,49 & KIMn & 0,44 & 29,26 & Simn & 0,06 & 29,04 \\
\hline FeilN & 1,31 & 26,34 & Zn'K & 1,60 & 22,29 & Znis & 11,64 & 18,98 \\
\hline NMn & 1,42 & 30,50 & $\mathrm{Mg} / \mathrm{Cl}$ & 0,41 & 15,41 & Cuí⿴囗十 & 0,45 & 39,35 \\
\hline$N Z n$ & 2,13 & 21,34 & cais & 3,21 & 17,76 & Felo & 3,36 & 46,02 \\
\hline PIK & 0,19 & 13,13 & $\mathrm{~B} / \mathrm{Ca}$ & 3,55 & 47,51 & Bimn & 0,63 & 42,35 \\
\hline CaiP & 2,32 & 13,94 & $\mathrm{Cu} / \mathrm{Ca}$ & 1,39 & 27,24 & $\mathrm{~B} / \mathrm{Zn}$ & 0,94 & 37,01 \\
\hline PiMg & 1,09 & 10,56 & FelCa & 9,92 & 30,61 & $\mathrm{Fel} \mathrm{Cu}$ & 7,26 & 22,31 \\
\hline PIS & 1,38 & 11,87 & Calmn & 0,19 & 32,82 & Cuimn & 0,25 & 22,40 \\
\hline $\mathrm{Bi} P$ & 7,82 & 34,82 & $\mathrm{Ca} / \mathrm{Zn}$ & 0,29 & 25,92 & CuiZn & 0,38 & 24,01 \\
\hline CuiP & 3,17 & 23,45 & $\mathrm{Mg} / \mathrm{S}$ & 1,29 & 19,39 & Feimn & 1,78 & 25,48 \\
\hline Feip & 22,88 & 30,36 & Bimg & 8,70 & 40,82 & Fel Zn & 2,75 & 29,60 \\
\hline PiMn & 0,08 & 33,60 & Cuimg & 3,49 & 28,46 & $\mathrm{Zn} / \mathrm{Mn}$ & 0,68 & 28,74 \\
\hline Zn'P & 8,47 & 18,43 & & & & & & \\
\hline
\end{tabular}

TABELA 5 - Valores de referência do DRIS (média e coeficiente de variação - CV) obtidos de amostras foliares e de grãos da cultivar Embrapa 59 coletadas em área manejada no sistema plantio direto na palha em Carambeí, PR.

\begin{tabular}{|c|c|c|c|c|c|c|c|c|}
\hline $\begin{array}{c}\text { Relaçẫo } \\
\text { nutricional }\end{array}$ & Média & $C V-\%$ & $\begin{array}{c}\text { Relaçẫo } \\
\text { nutricional }\end{array}$ & Média & $C V-\%$ & $\begin{array}{c}\text { Relaçẫo } \\
\text { nutricional }\end{array}$ & Média & $C V-\%$ \\
\hline PIN & 0,07 & 20,26 & Caik & 0,30 & 15,53 & Feimg & 26,37 & 32,16 \\
\hline KIN & 0,42 & 15,86 & $\mathrm{Mg} / \mathrm{K}$ & 0,13 & 14,81 & $M \mathrm{~g} m \mathrm{~m}$ & 0,11 & 31,10 \\
\hline Cail & 0,13 & 17,63 & $\mathrm{~K} / \mathrm{S}$ & 8,92 & 21,84 & $M g i z n$ & 0,08 & 20,78 \\
\hline $\mathrm{Mg} / \mathrm{N}$ & 0,05 & 15,86 & BIK & 2,90 & 19,51 & Bis & 25,62 & 26,20 \\
\hline Nis & 21,37 & 20,20 & CuIK & 0,48 & 24,60 & Cu/s & 4,20 & 22,59 \\
\hline$B I N$ & 1,20 & 18,43 & Felk & 3,35 & 33,47 & Feis & 29,21 & 31,87 \\
\hline CuIN & 0,20 & 25,45 & KiMn & 0,84 & 34,98 & Mn's & 11,51 & 31,90 \\
\hline FelN & 1,40 & 33,81 & $\mathrm{~K} I Z n$ & 0,68 & 23,40 & $\mathrm{siZn}$ & 0,08 & 21,93 \\
\hline NiMn & 2,04 & 39,80 & $\mathrm{Mg} / \mathrm{Ca}$ & 0,43 & 16,38 & $\mathrm{~B} / \mathrm{Cu}$ & 6,40 & 35,17 \\
\hline NiZn & 1,63 & 25,20 & cais & 2,67 & 19,93 & BiFe & 1,04 & 72,98 \\
\hline PIK & 0,16 & 17,79 & $\mathrm{~B} / \mathrm{Ca}$ & 9,80 & 26,14 & Mn'B & 0,47 & 33,72 \\
\hline $\mathrm{P} / \mathrm{Ca}$ & 0,53 & 17,07 & $\mathrm{Cu} / \mathrm{Ca}$ & 1,62 & 26,20 & $\mathrm{~B} / \mathrm{Zn}$ & 1,97 & 33,21 \\
\hline Pimg & 1,27 & 18,08 & Feica & 11,03 & 29,81 & $\mathrm{FelCu}$ & 7,19 & 34,55 \\
\hline Pis & 1,41 & 22,94 & Calmn & 0,25 & 31,89 & $\mathrm{Mricu}$ & 2,87 & 40,11 \\
\hline Bif & 19,05 & 34,20 & $\mathrm{Ca} / \mathrm{Zn}$ & 0,20 & 22,79 & CuiZn & 0,32 & 22,36 \\
\hline $\mathrm{PICU}$ & 0,35 & 30,25 & Mg/s & 1,12 & 21,10 & Felmn & 2,99 & 24,43 \\
\hline Feip & 20,78 & 24,63 & Biligg & 23,24 & 25,61 & Feizn & 2,16 & 27,15 \\
\hline PilMn & 0,13 & 29,52 & $\mathrm{Mg} / \mathrm{CH}$ & 0,28 & 26,87 & $\mathrm{Mn} / \mathrm{Zn}$ & 0,85 & 22,40 \\
\hline $\mathrm{P} / \mathrm{Zn}$ & 0,11 & 21,89 & & & & & & \\
\hline
\end{tabular}


MAEDA, S.; RONZELLI Jr., P. Valores de referência do dris para...

TABELA 6 - Valores de referência do DRIS (média e coeficiente de variação - CV) obtidos de amostras foliares e de grãos da cultivar Embrapa 59 coletadas em área manejada no sistema plantio convencional em Carambeí, PR.

\begin{tabular}{|c|c|c|c|c|c|c|c|c|}
\hline $\begin{array}{c}\text { Relaçẫo } \\
\text { nutricional }\end{array}$ & Média & $C V-\%$ & $\begin{array}{c}\text { Relaçẫo } \\
\text { nutricional }\end{array}$ & Média & $C V-\%$ & $\begin{array}{c}\text { Relaçẫo } \\
\text { nutricional }\end{array}$ & Média & $C V-\%$ \\
\hline NP & 17,03 & 18,92 & $\mathrm{KICa}$ & 2,71 & 11,27 & Feimg & 29,79 & 25,15 \\
\hline NiK & 3,06 & 19,77 & KIMg & 6,84 & 14,72 & Mrimg & 11,97 & 28,62 \\
\hline $\mathrm{NCa}$ & 8,22 & 20,62 & SiK & 0,14 & 24,29 & $M g / Z n$ & 0,08 & 24,60 \\
\hline NiMg & 20,82 & 24,02 & BiK & 4,32 & 19,07 & Bis & 33,18 & 31,85 \\
\hline NS & 23,31 & 30,23 & $\mathrm{KICU}$ & 2,44 & 35,92 & Sicu & 0,33 & 44,56 \\
\hline$B i N$ & 1,43 & 14,43 & Feik & 4,40 & 24,36 & Fels & 33,40 & 30,09 \\
\hline CuIN & 0,15 & 33,83 & KiMn & 0,61 & 30,21 & Simn & 0,08 & 33,99 \\
\hline FelN & 1,45 & 21,21 & $\mathrm{~K} I \mathrm{Zn}$ & 0,55 & 25,58 & SiZn & 0,07 & 26,00 \\
\hline NMn & 1,82 & 25,66 & $\mathrm{Mg} / \mathrm{Ca}$ & 0,40 & 7,89 & $\mathrm{~B} / \mathrm{Cu}$ & 10,40 & 38,61 \\
\hline$N Z n$ & 1,62 & 20,06 & sica & 0,37 & 21,47 & Fe'B & 1,03 & 24,48 \\
\hline KIP & 5,64 & 12,79 & $\mathrm{~B} / \mathrm{Ca}$ & 11,64 & 20,53 & $B / M n$ & 2,59 & 28,62 \\
\hline PiCa & 0,48 & 10,61 & $\mathrm{CalCu}$ & 0,91 & 38,35 & $\mathrm{~B} / \mathrm{Zn}$ & 2,32 & 24,97 \\
\hline PiMg & 1,22 & 10,71 & $\mathrm{Felca}$ & 11,77 & 22,80 & Felcu & 10,56 & 38,40 \\
\hline SiP & 0,76 & 18,48 & cailmn & 0,23 & 27,01 & Cuimn & 0,27 & 34,94 \\
\hline BiP & 24,12 & 18,88 & Caizn & 0,20 & 23,80 & Cuizn & 0,24 & 31,27 \\
\hline PiCu & 0,43 & 35,15 & Simg & 0,37 & 21,47 & Fejwn & 2,59 & 25,47 \\
\hline Felp & 24,50 & 23,03 & Bimg & 29,44 & 23,09 & Feizn & 2,30 & 19,04 \\
\hline PiMn & 0,11 & 26,57 & $\mathrm{Mg} / \mathrm{Cu}$ & 0,36 & 36,54 & Znimn & 1,14 & 23,98 \\
\hline$P I Z n$ & 0.10 & 20,86 & & & & & & \\
\hline
\end{tabular}

TABELA 7 - Valores de referência do DRIS (média e coeficiente de variação - CV) obtidos de amostras foliares e de grãos da cultivar Embrapa 59 coletadas em área manejada nos sistemas plantio direto na palha e convencional e da cultivar BR 37 manejada no sistema convencional, em Carambeí, PR.

\begin{tabular}{|c|c|c|c|c|c|c|c|c|}
\hline $\begin{array}{c}\text { Relaçẫo } \\
\text { nutricional }\end{array}$ & Média & $C V-\%$ & $\begin{array}{c}\text { Relaçẫo } \\
\text { nutricional }\end{array}$ & Média & $C V-\%$ & $\begin{array}{c}\text { Relaçẫo } \\
\text { nutricional }\end{array}$ & Média & $C V-\%$ \\
\hline PIN & 0,06 & 18,70 & CalK & 0,35 & 19,56 & $M g i F e$ & 0,04 & 56,08 \\
\hline KIN & 0,37 & 21,45 & $\mathrm{Mg} / \mathrm{K}$ & 0,14 & 18,93 & $M g / m n$ & 0,09 & 33,20 \\
\hline Cain & 0,13 & 18,71 & SiK & 0,13 & 22,87 & $M g i Z n$ & 0,09 & 27,80 \\
\hline $\mathrm{Mg} / \mathrm{N}$ & 0,05 & 18,91 & $\mathrm{~KB}$ & 0,38 & 63,59 & Sig & 0,05 & 65,60 \\
\hline SIN & 0,05 & 20,33 & Cu'K & 0,50 & 31,74 & $\mathrm{SiCu}$ & 0,28 & 40,33 \\
\hline$B N$ & 1,17 & 33,65 & $\mathrm{KFe}$ & 0,30 & 60,74 & SiFe & 0,04 & 57,54 \\
\hline CuIN & 0,18 & 29,64 & KMn & 0,69 & 40,44 & Simn & 0,08 & 38,46 \\
\hline FelN & 1,41 & 28,20 & $\mathrm{~K} Z \mathrm{Zn}$ & 0,62 & 25,53 & $S i Z n$ & 0,08 & 24,39 \\
\hline MriN & 0,60 & 33,48 & $\mathrm{Mg} / \mathrm{Ca}$ & 0.41 & 13,98 & Cu'B & 0,09 & 74,61 \\
\hline$Z n / N$ & 0,62 & 23,64 & $\mathrm{SiCa}$ & 0,37 & 20,66 & Felo & 1,49 & 72,84 \\
\hline PIK & 0,17 & 16,29 & $\mathrm{Ca}^{\prime} \mathrm{B}$ & 0,14 & 86,19 & Mr'B & 0,68 & 95,23 \\
\hline $\mathrm{PiCa}$ & 0,50 & 16,41 & $\mathrm{Ca}{ }^{\prime} \mathrm{Cu}$ & 0,78 & 37,72 & Zni & 0,63 & 57,01 \\
\hline $\mathrm{Mg} P \mathrm{P}$ & 0,84 & 15,39 & CalFe & 0,10 & 54,85 & Cu'Fe & 0,15 & 79,09 \\
\hline SiP & 0,75 & 19,73 & Calmn & 0,23 & 31,58 & Cu'mn & 0.32 & 40,53 \\
\hline Pig & 0,07 & 69,48 & $\mathrm{Ca} Z \mathrm{Zn}$ & 0,22 & 27,94 & Cu'Zn & 0,30 & 30,44 \\
\hline PiCu & 0,38 & 34,06 & simg & 0,37 & 20,66 & MriFe & 0,46 & 51,13 \\
\hline PiFe & 0,05 & 58,24 & $\mathrm{Mg} / \mathrm{B}$ & 0,06 & 83,72 & Feizn & 2,31 & 26,38 \\
\hline PIMn & 0,11 & 32,68 & $\mathrm{Mg} / \mathrm{Cu}$ & 0,31 & 34,64 & Znimn & 1,11 & 30,68 \\
\hline $\mathrm{P} i \mathrm{Zn}$ & 0,11 & 22,95 & & & & & & \\
\hline
\end{tabular}


MAEDA, S.; RONZELLI Jr., P. Valores de referência do dris para...

\section{CONCLUSÕES}

Os valores de referência do DRIS, obtidos a partir das amostras da cultivar Embrapa 59 no sistema plantio direto na palha e sistema plantio convencional (SPC) e da cultivar BR 37 no SPC, proporcionam estimativas diferentes para os índices de diagnose do DRIS resultando em diagnósticos distintos.

\section{REFERÊNCIAS BIBLIOGRÁFICAS}

1. BATAGLIA, O.C.; DECHEN, A.R. Critérios alternativos para diagnose foliar. In: SIMPÓSIO AVANÇADO DE QUÍMICA E FERTILIDADE DO SOLO, 1., 1986, Piracicaba. Anais... Campinas: Fundação Cargill, 1986. p.115-136.

2. BEAUFILS, E.R. Diagnosis and Recommendation Integrated System (DRIS); a general scheme for experimentation and calibration based on principles develop from research in plant nutrition. Pietermaritzburg: University of Natal, 1973. 132p. (Soil Science Bulletin, 1).

3. BEVERLY, R.B.; SUMNER, M.E.; LETZSCH, W.S.; PLANCK, C.O. Foliar diagnosis of soybean by DRIS. Communications in Soil Science and Plant Analysis, New York, v.17, p.237-256, 1986.

4. BORKERT, C.M.; YORINORI, J.T.; CORREA-FERREIRA, B.S.; ALMEIDA, A.M.R.; FERREIRA, L.P.; SFREDO, G.J. Seja o doutor da sua soja. Informações Agronômicas, Piracicaba, n.66, jun. 1994. Arquivo do Agrônomo, Piracicaba, n.5, p.1-16, jun. 1994.

5. COSTA, J.A.; MARQUEZAN, E. Características dos estádios de desenvolvimento da soja. Campinas: Fundação Cargill, 1982. 30p.

6. ELWALI, A.M.O.; GASCHO, G.J. Soil testing, foliar analysis and DRIS as guides for sugarcane fertilization. Agronomy Journal, Madison, v.76, p.466-470, 1984

7. EMBRAPA SOJA. Recomendações técnicas para a cultura da soja no Paraná - 1999/2000. Londrina, 1999. 236p. (Embrapa Soja. Documentos, 131).

8. KEOGH, J.L.; SABBE, W.B.; CAVINESS, C.E. Nutrient concentration of selected soybean cultivars. Communications in Soil Science and Plant Analysis, New York, v.3, p.29-36, 1972.

9. LEANDRO, W.M. Sistema Integrado de Diagnose e Recomendação (DRIS) para a cultura da soja (Glycine max L. Merril) na região de Rio Verde-GO. Goiânia: 1998. 157f. Tese (Doutorado em Agronomia, Produção Vegetal) - Universidade Federal de Goiás.

10. MALAVOLTA, E.; VITTI, G.C.; OLIVEIRA, S.A. de. Avaliação do estado nutricional de plantas: princípios e aplicações. Piracicaba: Potafós, 1997. $319 \mathrm{p}$.

11. MARTINEZ, H.E.P.; CARVALHO, J.G.; SOUZA, R.B. de. Diagnose foliar. In: RECOMENDAÇÕES para o uso de corretivos e fertilizantes em Minas Gerais - $5^{a}$ aproximação. Viçosa: Comissão de Fertilidade do Solo do Estado de Minas Gerais, 1999. p.143168.

12. MASCARENHAS, H.A.A.; NEPTUNE, A.M.L.; MURAOKA, T.; BUSILANI, E.A.; HIROCE, R. Absorção de nutrientes por cultivares de soja. Revista Brasileira de Ciência do Solo, Campinas, v.4, p.92-96, 1980.

13. SCHUTZ, C.J.; VILLIERS, J.M. Foliar diagnosis and fertilizer prescription in forestry - the DRIS system and its potential. South African Forestry Journal, Pretoria, v.141, p.6-12, 1987.

14. SUMNER, M.E. Interpretation of foliar analysis for diagnostic purposes. Agronomy Journal, Madison, v.41, p.343-348, 1979.

15. WALWORTH, J.L.; SUMNER, M.E. The Diagnosis and Recommendation Integrated System (DRIS). Advances in Soil Science, New York, v. 6, p. 149-188, 1987.

16. WALWORTH, J.L., WOODWARD, H.J., SUMNER, M.E. Generation of corn tissue norms from a small, high-yield data base. Communications in Soil Science and Plant Analysis, New York, v.19, p.563-577, 1988. 